\title{
Rankin Inlet PolarDARN radar observations of duskward moving Sun-aligned optical forms
}

\author{
A. Koustov ${ }^{1,2}$, K. Hosokawa ${ }^{3}$, N. Nishitani ${ }^{2}$, T. Ogawa ${ }^{2}$, and K. Shiokawa ${ }^{2}$ \\ ${ }^{1}$ Institute of Space and Atmospheric Studies, University of Saskatchewan, 116 Science Place, Saskatoon, S7N 5E2 Canada \\ ${ }^{2}$ Solar-Terrestrial Environment Laboratory, Nagoya University, Nagoya, Japan \\ ${ }^{3}$ University of Electro-Communications, Tokyo, Japan
}

Received: 4 May 2008 - Revised: 15 July 2008 - Accepted: 5 August 2008 - Published: 12 September 2008

\begin{abstract}
On 15 February 2007, several duskward moving sun-aligned (SA) auroral forms have been observed by the all-sky camera at Resolute Bay, Nunavut (Canada). Concurrent observations with the Rankin Inlet (RANK) PolarDARN HF radar within the field-of-view of the camera showed signatures of moving auroral forms in all signal parameters with the most remarkable effects being the echo power drop and velocity reversal as the arc reached a specific radar beam/gate. Spatial and temporal variations of the velocity in the vicinity of the SA form are investigated. It is shown that the form-associated convection reversal was located poleward (duskward) of the global-scale convection reversal associated with the dawn cell of the large-scale convection pattern. Thus, the RANK radar was monitoring the polar cap portion of the global-scale convection pattern and its transition from the IMF $B_{y}<0$ to the $B_{y}>0$ situation. Magnetic perturbations associated with the SA form passing the zenith of several magnetometers are investigated. It is shown that although magnetometer signatures of the moving form were clear, the convection pattern derivation from magnetometer records alone is not straightforward.
\end{abstract}

Keywords. Ionosphere (Electric fields and currents; Ionosphere-magnetosphere interactions; Polar ionosphere)

\section{Introduction}

Aurora is well known to occur poleward of the auroral oval latitudes, inside the polar cap, all the way to the magnetic pole (e.g. Zhu et al., 1997; Hultqvist, 2007). It has been seen in a variety of forms and intensity. One of the well established classes of the high-latitude aurora is the so called sun-aligned (SA) arcs. SA arcs are relatively narrow bands of luminosity stretching in the direction roughly towards the

Correspondence to: A. Koustov

(sasha.koustov@usask.ca)
Sun through the entire field of view of a ground-based all-sky camera, a typical instrument for SA observations. The arcs occur predominantly for the northward-oriented interplanetary magnetic field (IMF), $B_{z}>0$. They are often seen as "detaching" from the auroral oval on its either dusk or dawn side and moving through the central polar cap and reaching sometimes the pole and even further to the opposite side of the polar cap (Zhu et al., 1997). In this sense, SA arcs are similar to more intense auroral displays known as the thetaaurora that is not only more intense but also more global and, for these reasons, identifiable from satellite images. The direction of the SA motion, dawnward or duskward, was found to be strongly controlled by the IMF $B_{y}$ component (Valladares et al., 1994; Zhu et al., 1997; Kullen et al., 2002): in the Northern Hemisphere, the dawn (dusk-) sector arcs move towards dusk (dawn) for IMF $B_{y}>0\left(B_{y}<0\right)$.

Although many morphological properties of SA arcs have been identified (e.g. Valladares et al., 1994; Kullen et al., 2002), the physical processes leading to their onset and subsequent evolution are not well understood (Zhu et al., 1997). Part of the problem is that the acquired data are limited and controversial in many aspects. On the theoretical side, proposed models of SA formation (e.g. Sojka et al., 1994; Zhu et al., 1997) require a number of input parameters that are not well known in individual cases. One of these is the globalscale plasma convection pattern. Information on plasma convection is not readily available as convection measurements have been limited to either one direction, as for the case of an incoherent scatter radar (e.g. Robinson et al., 1987; Valladares and Carlson, 1991; Gallagher, 1997), or to one trajectory (line) as for the case of ion drift measurements on satellites (e.g. Carlson et al., 1988). The network of SuperDARN HF radars has significantly improved capabilities of the space science community in monitoring the global-scale plasma convection pattern, and several recent publications have contributed to understanding of theta auroras (Chang et al., 1998; Milan et al., 2005; Liu et al., 2005; Eriksson et al., 2006).

Published by Copernicus Publications on behalf of the European Geosciences Union. 


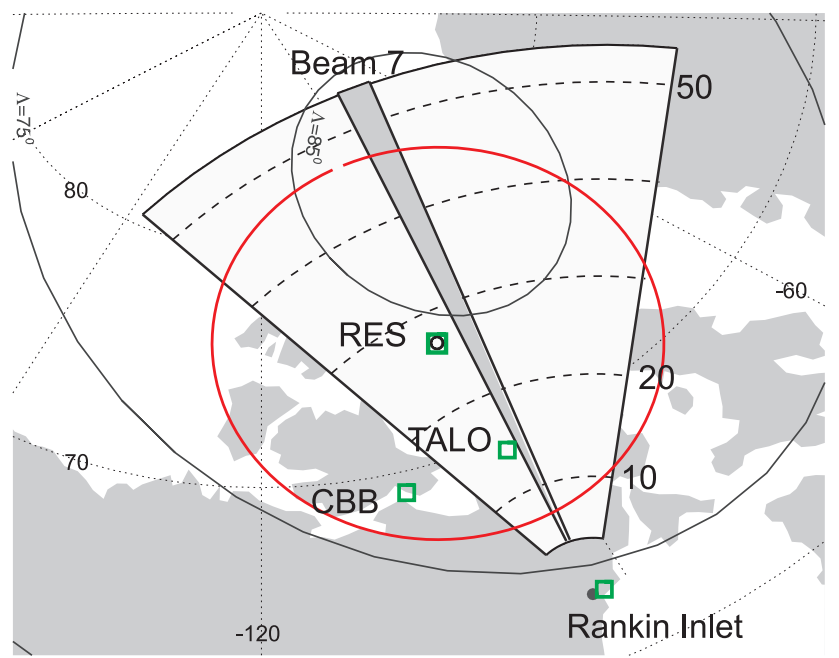

Fig. 1. Field of view (FoV) of the Rankin Inlet (RANK) PolarDARN radar between ranges of 400 and $2600 \mathrm{~km}$ and location of ground-based magnetometers data from which are used in the study. The dashed lines indicate radar ranges according to the gate number $(10,20,30,40,50)$. A shaded beam-like area within the radar FoV is the position of beam 7. Red circle represents the FoV of all-sky camera (for the off-zenith angles of $<75^{\circ}$ ) at Resolute Bay (RES) for the height of $250 \mathrm{~km}$.

For the case of SA arcs, attempts have not been so successful. One of the reasons is, perhaps, a lack of radar data in close vicinity of the optical forms where HF radio waves are strongly refracted/reflected (Uspensky et al., 1994) or simply absorbed. In addition, to observe high-latitude echoes associated with polar cap arcs, the SuperDARN radars, that are located at the equatorward edge of the auroral oval, would have to rely on echoes received through $1 \& 1 / 2$ hop propagation mode (ranges of $>2500-3000 \mathrm{~km}$ ); such echoes are very sensitive to the aurora presence as enhanced electron density can change significantly the radar wave path.

Recently installed HF PolarDARN radar (part of the SuperDARN network) at Rankin Inlet (RANK) monitors the ionosphere at latitudes above $75^{\circ}$ MLAT. The field-of-view (FoV) of the RANK radar covers latitudes up to and even over the magnetic pole, including an area around the Resolute Bay (RES) where several optical instruments are set to monitor high-latitude auroras; among these is the OMTI all-sky camera run by the Solar-Terrestrial Environment Laboratory (STEL), Nagoya University (Shiokawa et al., 1999). RES is located at relatively short ranges from RANK $(\sim 1300 \mathrm{~km})$; thus, the RANK radar - RES OMTI camera is a very powerful combination for studies of the polar cap physics, including SA arcs.

In this paper we investigate one case of SA arc observations using the RANK radar and RES all-sky camera with a primary goal to establish radar signatures of SA arc and then to investigate plasma flows associated with such an arc.

\subsection{Geometry of observations}

Figure 1 shows the FoV of the Rankin Inlet PolarDARN/SuperDARN radar and the FoV of the all-sky camera at Resolute Bay (for the off-zenith angle of $75^{\circ}$ ). Radar range ticks are made for gates 10, 20, 30, 40 and 50 (ranges 630, 1080, 1530 and $1980 \mathrm{~km}$ ). Within the radar FoV we indicate the position of the radar beam 7 data from which will be discussed in more detail. Also shown are the locations of several ground magnetometers. One can see that the RES camera is optimal for joint work with the RANK radar for radar range gates 25-30, corresponding to radar slant ranges of $\sim 1300$ $1500 \mathrm{~km}$. Although these are typical ranges for $F$ region echo detection in the auroral zone (e.g. Danskin et al., 2002), for the magnetically higher latitude radars (such as Halley and Rankin Inlet), the echoes are more frequent at shorter ranges, $<1200 \mathrm{~km}$ for the Rankin Inlet radar. This experimental finding, realized after the RANK radar was installed, unfortunately, reduces the chances for co-located optical-radar studies.

In this paper we consider one event of Sun-aligned arc monitoring by the RANK radar on 15 February 2007. Over this day, a significant amount of joint data was collected so that several phenomena can be investigated. We focus on relatively short period of $\sim 30 \mathrm{~min}$ in duration between 07:50 and 08:20 UT. During this period, clear SA form appeared at the eastern edge of the RES camera FoV, it then moved duskward (to the West), reached the RES zenith and, after making several stops, moved to the western part of the RES camera FoV and eventually disappeared.

\section{All-sky images of the sun-aligned arc}

Figure 2 shows three RES OMTI raw camera images (in red line of $630 \mathrm{~nm}$ ) illustrating the event under investigation. At 07:56 UT, near the beginning of the event, an optical form is seen stretching in the north-south direction. At 08:10 UT, it is located in the zenith of RES. One clearly recognizes that, at this time, the luminosity band consists of several forms with two strongest ones at its eastern and western edges so that the form can be said to consist of two arcs oriented towards the Sun. The overall width of the luminosity band is of the order of $\sim 100 \mathrm{~km}$ if one assumes the luminosity heights to be $250 \mathrm{~km}$. By $08: 20 \mathrm{UT}$, the luminosity band passed the RES zenith and is seen as a single sun-aligned form. We should say that the double-arc structure of the form was not recognizable in optical images prior to $\sim 07: 58$ UT and after $\sim 08: 16$ UT. The splitting of a single optical form in the course of the event is consistent with the radar data that will be discussed later. This means that the optical form under discussion was not only relatively broad, but also was changing in configuration over the event duration. Despite this fact, we shall use interchangeably the terms SA arc and SA form. 

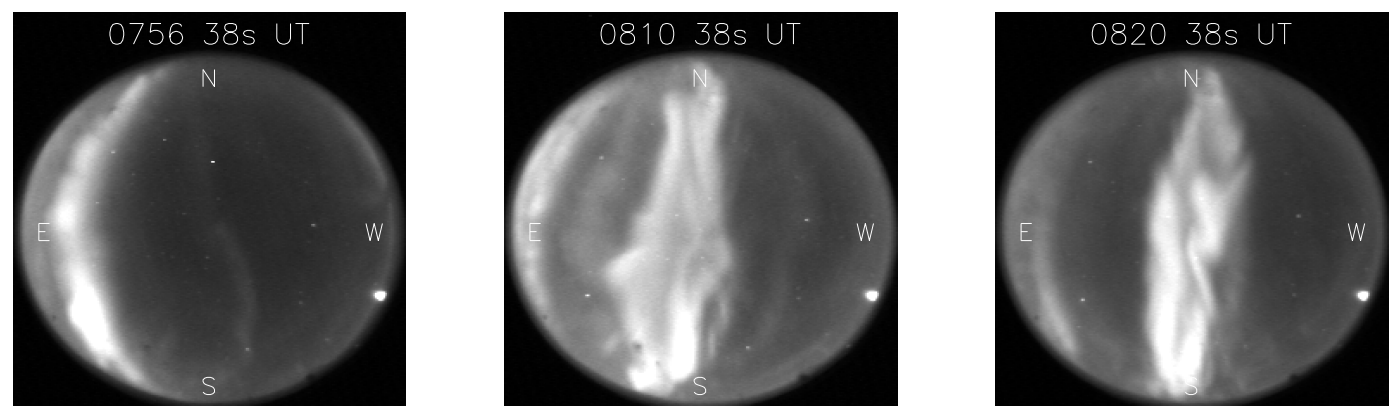

Fig. 2. Three all sky images of sun-aligned auroral forms recorded by the OMTI camera at a wavelength of $630 \mathrm{~nm}$ at Resolute Bay on 15 February 2007.

\section{General conditions on 15 February 2007 and onset of sun-aligned arc}

15 February 2007 was a relatively quiet day with the $K_{p}$ index of $2 \circ$ between 06:00 and 09:00 UT. The low activity is expected from the IMF conditions as the $B_{z}$ component was positive over a significant portion of this period, Fig. 3.

In Fig. 3, the ACE measurements of the IMF (with a time delay of 57 min to account for the disturbance propagation from the satellite location to the midnight sector of the high-latitude ionosphere) are presented. Shaded box indicates period of the ground-based measurements under discussion. There is always some uncertainty with the estimate of the time shift for the ACE measurements. For the considered event, the solar wind speed was quite high, $\sim 700 \mathrm{~km} / \mathrm{s}$, which may introduce a significant error in the delay estimates. In addition, the event occurred on the nightside which imply even more uncertainty. In selecting of the 57-min delay, we used the widely accepted opinion that motion of SA arcs respond to a quick change in the IMF $B_{y}$ while $B_{z}$ is still being positive. The considered interval matches this criterion well. Additional testing of the delay selection has been done by considering optical signatures during the period of 05:00-09:00 UT with similar IMF conditions. It was found that four other duskward moving SA-like events occurred. The beginnings of all of these are marked by dotted lines in Fig. 3: $\sim 05: 30$ UT $(\sim 15 \mathrm{~min}$ in duration, the forms were only seen at the edge of the camera FoV), $\sim 06: 05$ UT (event lasting $\sim 30 \mathrm{~min}$ ), $\sim 06: 50 \mathrm{UT}$ (short event with progression at the equatroward edge of the FoV) and 09:25 UT (event lasting $\sim 30 \mathrm{~min}$ ). For these periods, the IMF conditions satisfy also the above criterion. In addition, over the main event that we consider in this study, there were several activations of duskward motions. The times for these activations are denoted by the vertical solid lines in Fig. 3; these times also match the criterion.

It should be noted that the onset of the SA events was preceded by substorm activity in the Canadian sector of Arctic as indicated by magnetic bays recorded by the CARISMA magnetometers between 02:00 and 05:00 UT with the Pi2 onsets around 02:00 UT and 02:50 UT. The RES camera

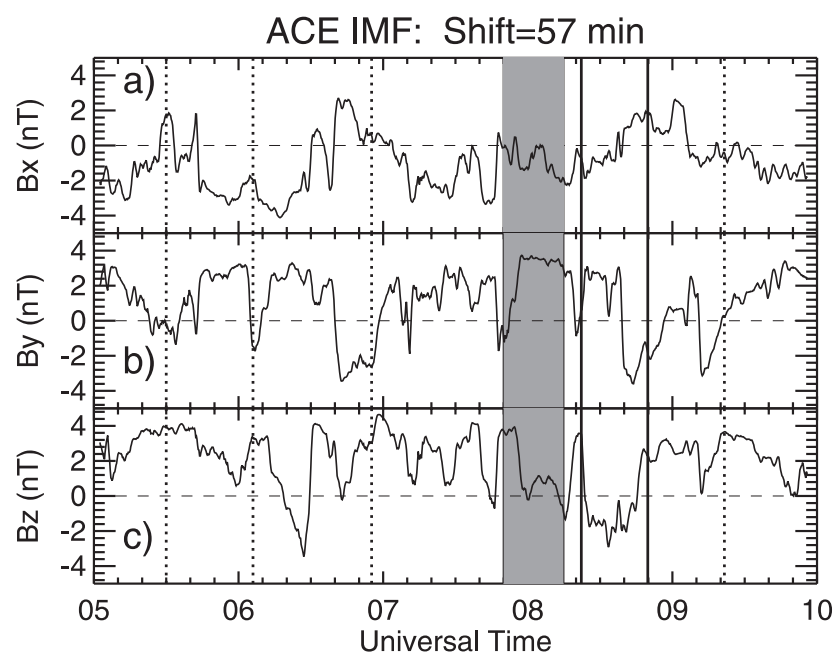

Fig. 3. Interplanetary magnetic field (IMF) $x, y$ and $z$ components in the GSM coordinate system according to the ACE satellite measurements on 15 February 2007 between 05:00 and 09:00 UT. The data were shifted by $57 \mathrm{~min}$. Shaded box correspond to the period during which SA forms were observed by the RES all-sky camera. Vertical solid lines indicate moments of easily recognizable start in the duskward progression of the SA forms.

was detecting poleward expanding aurora starting from about 03:00 UT. The active aurora did not reach very high-latitudes, such as the RES location, but was quite bright, lasting up to about 05:00 UT and showing several eastward and westward progressions. Occurrence of morning SA arcs after substorm activity in the midnight sector is then consistent with the results of Shiokawa et al. (1997).

\section{HF radar signatures of the sun-aligned arc}

Figure 4a shows the SA form in the MLT-MLAT coordinates; this is the same image as the one presented in the first frame of Fig. 2. The mapping is done by assuming the luminosity height of $250 \mathrm{~km}$ (630 nm filter data). The edges of the arc are enhanced by a ragged line; the western line corresponds 


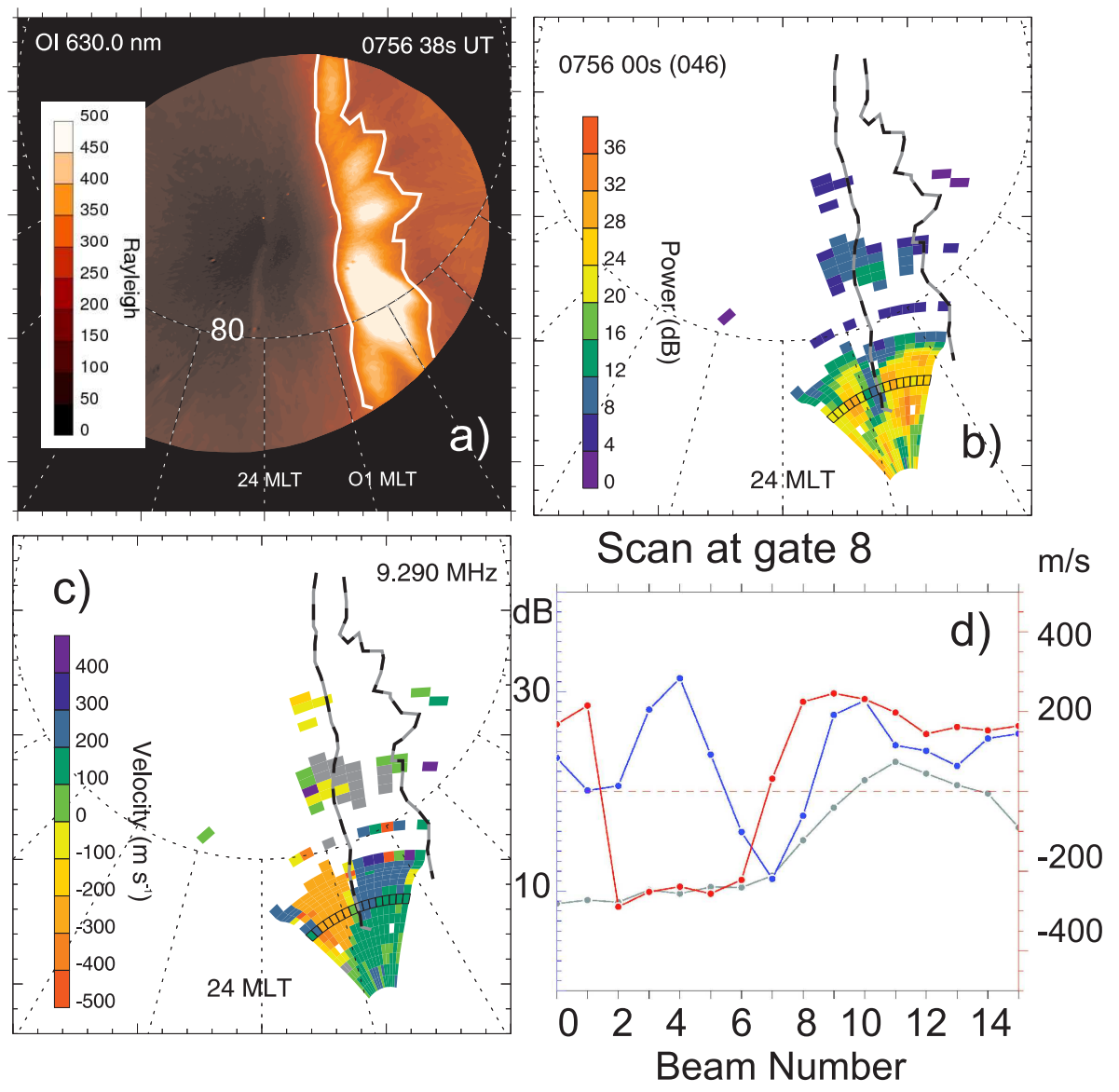

Fig. 4. (a) Auroral luminosity observed by the RES OMTI camera at 07:56:38 UT in MLT-MLAT coordinates. The locations of the arc edges are shown by white ragged line. The side closer to the RES location in the center (the duskward side) corresponds to the arc's upper part); (b) Rankin Inlet radar echo power map and the location of the luminosity edges as presented in panel (a); (c) Rankin Inlet radar echo velocity map (positive: toward the radar) and location of the luminosity edges as presented in panel (a); (d) Luminosity (black), echo power (blue) and velocity (red) variations with the beam number in gate 8 (range of $540 \mathrm{~km}$ ). Location of gate 8 is indicated on panels (b) and (c) by dashed lines.

to the bottom part of the luminosity at the F region heights. One can recognize sun-alignment of the luminosity band as it stretches through the entire FoV of the camera towards 12:00 MLT tick. The form is not homogenous; this was typical for other frames as well, especially towards the end of the event.

Figure $4 \mathrm{~b}$ shows the RANK echo power distribution for the scan that starts at 07:56 UT. Clear power minimum in central beams (blue color) is identifiable. This minimum spatially coincides with the duskward edge of the luminosity band. This edge of the optical form corresponds to the upper part of the luminosity, and it would mark the location of the arc if it were a thin structure at the $\mathrm{F}$ region heights.

Figure $4 \mathrm{c}$ shows the RANK velocity map. The duskward edge of the luminosity band collocates with an area of sharp velocity reverse from large negative (away from the radar) values in the western part of the map (low number beams) to large positive (toward the radar) values in the eastern part of the map (high number beams). The entire luminosity band corresponds to positive velocity, i.e. to antisunward plasma flow.

The above features in the mutual distribution of the auroral luminosity, echo power and velocity are quantitatively illustrated in Fig. 4d where these parameters are plotted against the radar beam number for gate 8 (slant range of $540 \mathrm{~km}$ ). These data correspond to one azimuthal scan at one range. The power drop associated with the duskward edge of the luminosity occurs in beam 7 , it is $\sim 20 \mathrm{~dB}$. Beam 7 almost corresponds to the direction in which the velocity changes its polarity; the velocity reverse occurs somewhere in between beams 6 and 7. Such sharp power drop and velocity reverse at the duskward edge of the luminosity band have been identified for almost all other scans during the entire period under consideration; these are the strongest signatures of SA arc in the HF radar data. To illustrate further these features, we present in Fig. 5 a sequence of echo power and velocity 

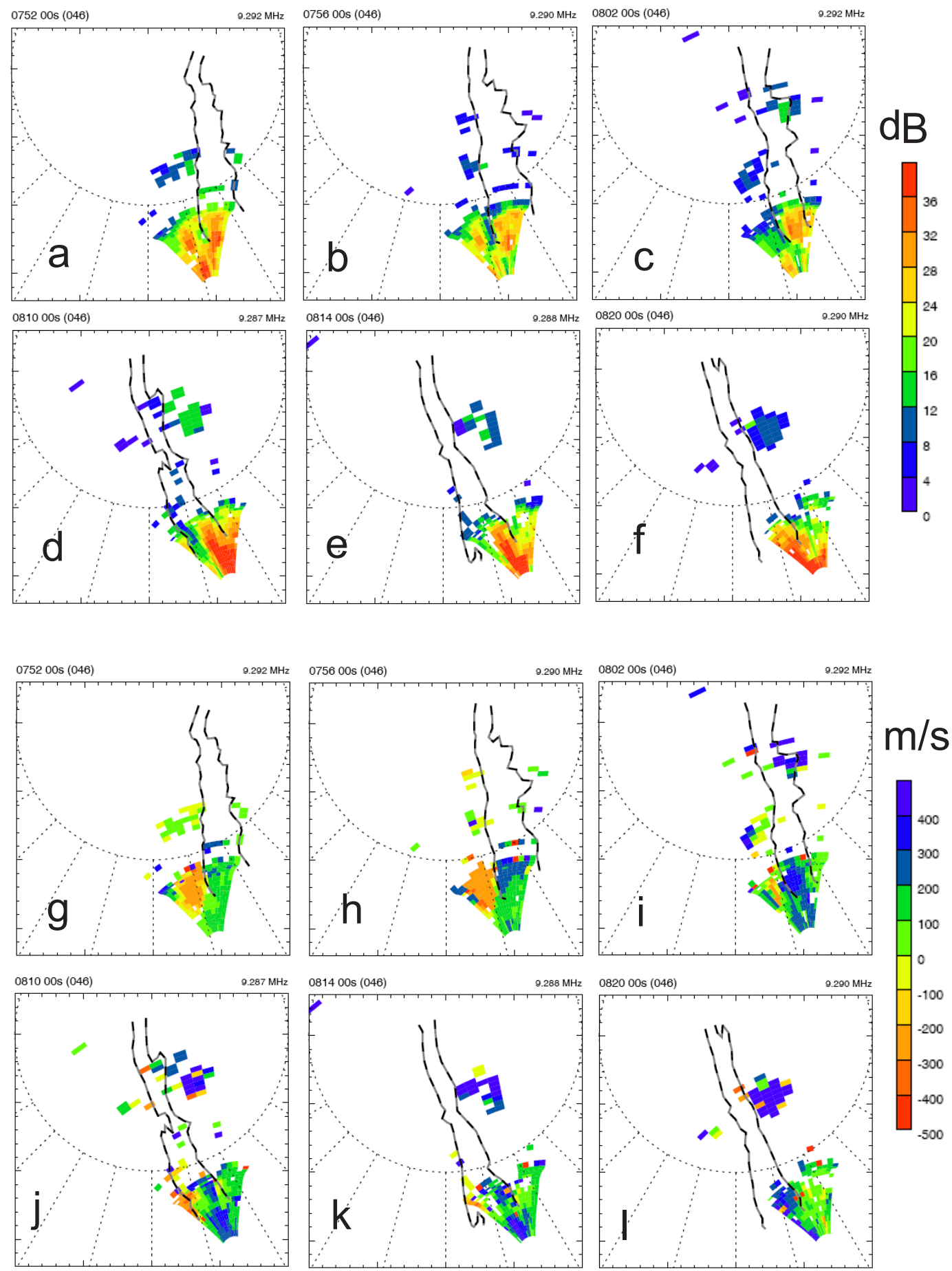

Fig. 5. Rankin Inlet HF (a-f) echo power and (g-l) velocity maps (positive: toward the radar) and locations of the auroral luminosity at closest time for the entire event (07:50-08:20 UT).

maps, together with the locations of the luminosity band over the entire period under consideration (07:50-08:20 UT). One can clearly recognize that the arc has been fast moving westward/duskward; our estimates show that the velocity of this motion was $\sim 100-200 \mathrm{~m} / \mathrm{s}$ (the form slowed down to- wards the end of the event). The echo power drop and the velocity reversal are shifting westward synchronously with the luminosity band. The exception is the last two frames, corresponding to the time when the arc was located westward of the RES zenith. We note that the eastern edge of 


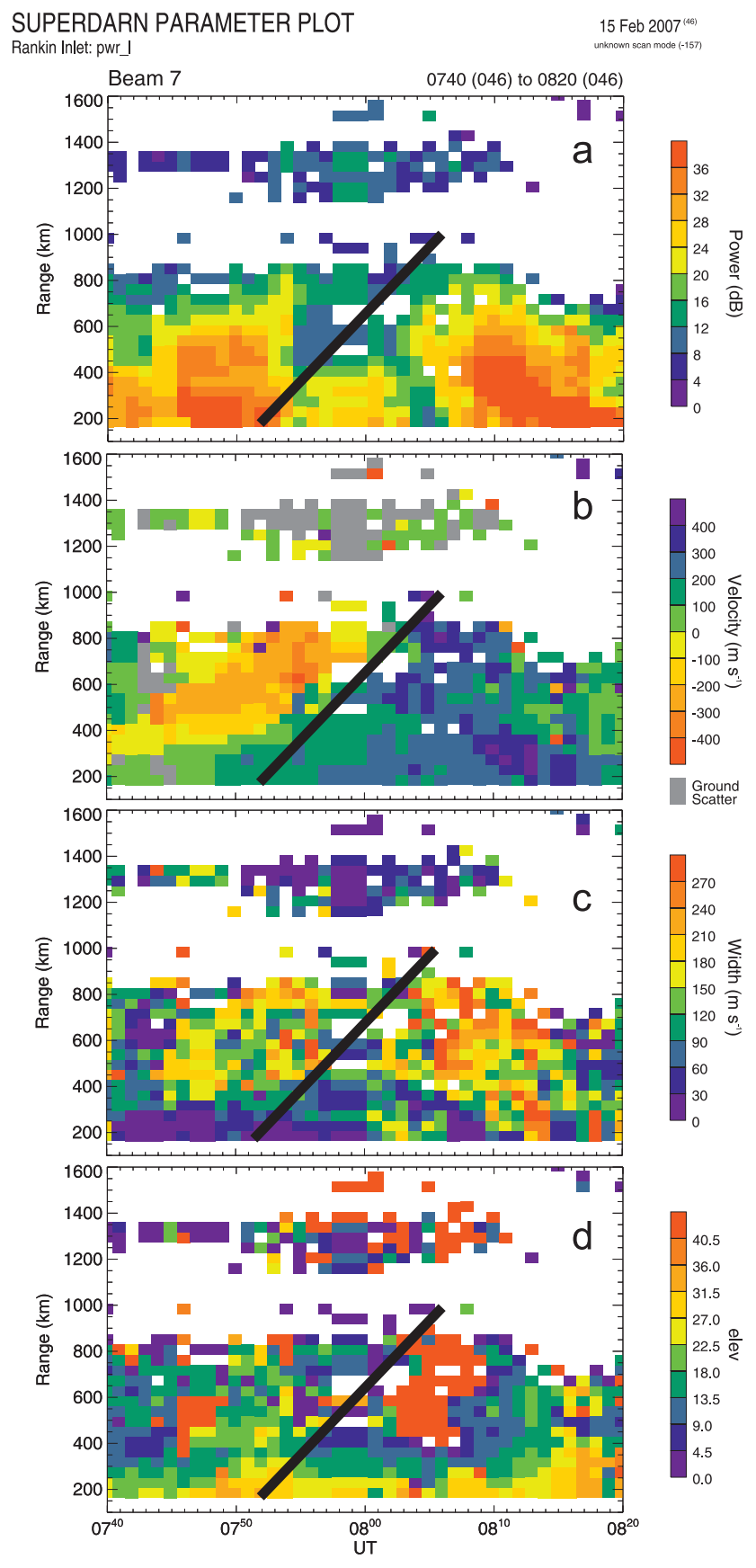

Fig. 6. Echo power, velocity, spectral width and elevation angle according to RANK measurements in beam 7 between 07:40 and 08:20 UT. Vertical sloped line is a marker introduced for convenience of the comparison.

the luminosity band does not reflect the width of the optical form; the luminosity here is accumulated from various heights that are impossible to establish. These last frames are presented to show that as the SA form moves away, both the echo power and velocity drop in magnitude (probably to the background levels) to the east from the optical form.

\section{Radar observations in a fixed beam}

Since the SA form was crossing the radar FoV from the East to the West at only small azimuthal angle to the central RANK radar beams, another way of monitoring its motion is to look at the data obtained in a fixed beam and investigate temporal variations of echo parameters. This would be similar to measurements with a single-beam incoherent scatter radar whose beam is fixed in certain direction. Certainly, for the case of an HF radar, the spatial resolution is much worse that makes measurements more ambiguous, but these are still informative.

Figure 6 shows temporal variations of the echo power, velocity, spectral width and elevation angle in the radar beam 7 . For convenience of inter-comparison between various panels of this diagram, we introduced a marker, the sloped line; to some extent, this line reflects the position of the luminosity band's duskward edge at various ranges and times, although imprecisely.

According to Fig. 6, echoes were observed at relatively short ranges of $<900 \mathrm{~km}$. The drop in echo power was detected during $\sim 8-10 \mathrm{~min}$ as the optical form was crossing the beam. The drop was not strong at ranges $<400 \mathrm{~km}$; in fact two different drops are recognizable, one at $\sim 07: 55$ UT and another one at $\sim 08: 04$ UT. We should mention that optical measurements at these short radar ranges were not available because of the limitations of the RES camera FoV. Another circumstance is that, at these short ranges, the echoes were very likely being received from the $\mathrm{E}$ region heights. Indeed, the electron density inside the polar cap arc can reach values of $\sim 2 \times 10^{5} \mathrm{~cm}^{-3}$ (Robinson et al., 1987), which is sufficient to strongly bend radio waves even at short ranges and large geometric aspect angles (e.g. Koustov et al., 2002). Additional argument for the above opinion comes from the elevation angle measurements presented on the bottom panel. One can clearly see that the elevation angle steadily decreases at farther ranges, as one expects if the echoes are coming from a fixed height, which can only be the E region height at such short ranges. Also, this trend is in contrast to elevation angle increase with range at farther ranges, beyond $\sim 300 \mathrm{~km}$.

In Fig. 6b, one can see that the echo power drop coincides well with the velocity reversal at various ranges. The velocity reversal seems better characterizing the arc passage and thus is a more convenient parameter for arc monitoring than the echo power. Our analysis for the $\sim 06: 00$ and 09:30 UT events showed that signatures in the velocity are still present whereas they are not obvious in the power. No change in velocity polarity occurred at short ranges $<400 \mathrm{~km}$ once again indicating that perhaps the SA arc was not extended so much in the equatorward direction and that perhaps the echoes here were coming from the E region as the velocity of such echoes can be different, even of opposite polarity, from the velocity of $\mathrm{F}$ region echoes (Makarevich et al., 2004).

Figure $6 \mathrm{c}$ shows the spectral width changes during the passage of the SA form. Although the variability of this 


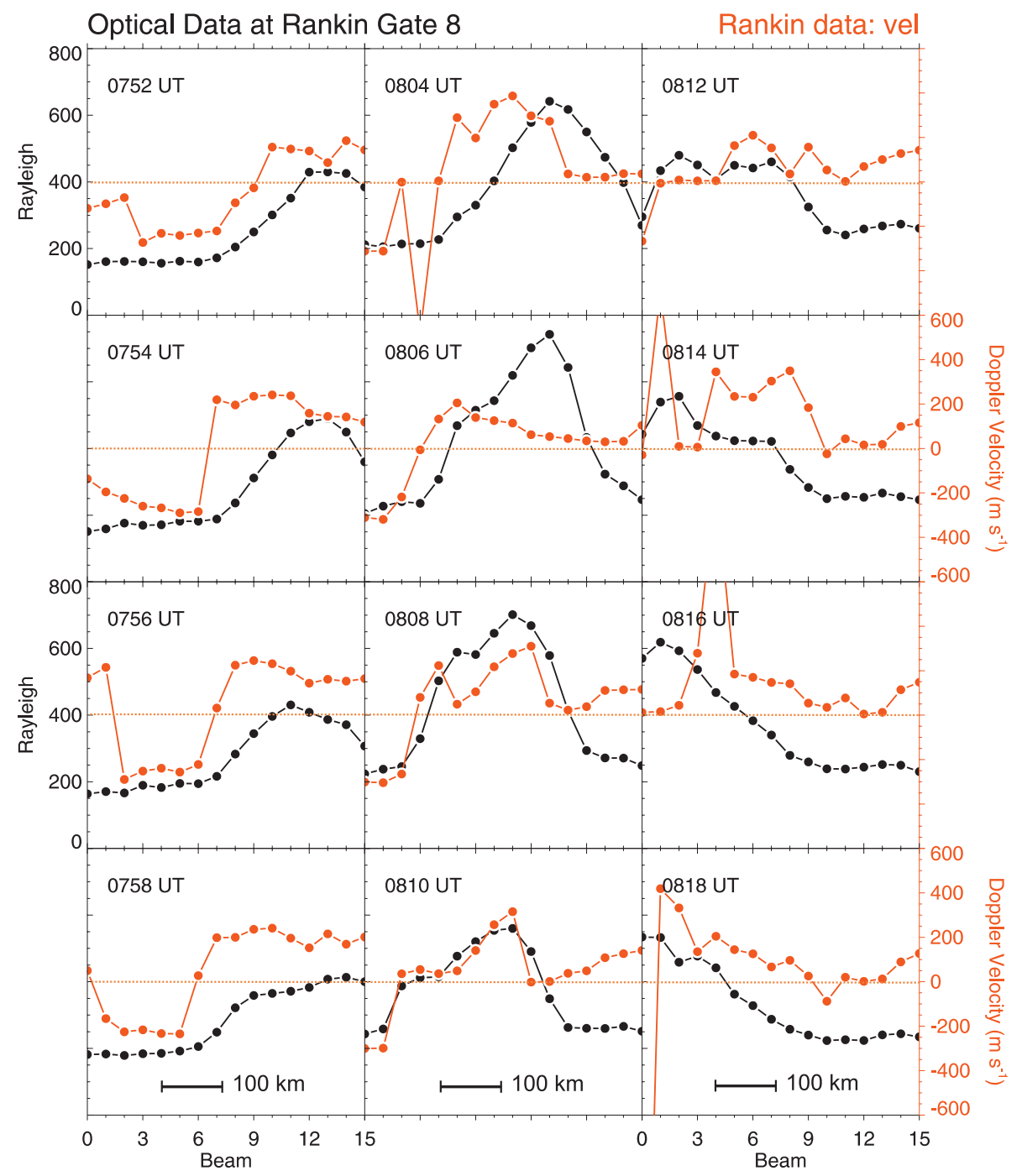

Fig. 7. RANK radar velocity in gate 8 (red) for a number of selected radar azimuthal scans between 07:52 and 08:20 UT. Black line is the luminosity intensity in radar gate 8 for every beam position. A scale bar at the bottom panels is a 100-km distance in the azimuthal direction (along a circle) at the radar range of $540 \mathrm{~km}$.

parameter is significant, it is safe to say that the echoes had increased spectral width in the vicinity of the SA form, near its both duskward and dawnward edges. The width is somewhat larger in the wake of the moving form, and this feature can be one of the signatures of the SA arc.

Finally, Fig. 6d shows another obvious signature of SA form crossing the RANK beam, namely strongly enhanced elevation angles on the form's dawnside. High elevation angles imply that the echoes are received here from larger heights. This can occur if the electron density is enhanced in the wake of it, at the F region heights. Such an effect has been predicted in a theoretical model of SA arc formation by Crain et al. (1993) and the presented HF observations can be considered as a confirmation of this theory.

\section{Radar data in gate 8 for selected scans}

Overall, the data presented in Figs. 5 and 6 illustrate the fact that the radar signatures of the SA optical forms moving across the polar cap can be found by looking at routine SuperDARN data plots alone. Now we investigate the details of the velocity distribution in the vicinity of SA form as plasma convection associated with these forms is one of the key questions of the polar cap electrodynamics (Zhu et al., 1997).

Figure 7 presents radar velocity data (red line) collected over a number of scans for the period under study. Measurements in one gate, number 8 (range of $540 \mathrm{~km}$ ), are considered as these were continuous over the entire event. We also show in Fig. 7 the luminosity intensity (black line) corresponding to the same gate for each beam position. The 


\section{OMTI ASI PARAMETER PLOT}

RSB vs RKN (emission height: $250 \mathrm{~km})$ : abs-data

\section{Feb $2007^{104}$}

filter id: $\mathrm{C62}$
(exposure $30 \mathrm{sec}$

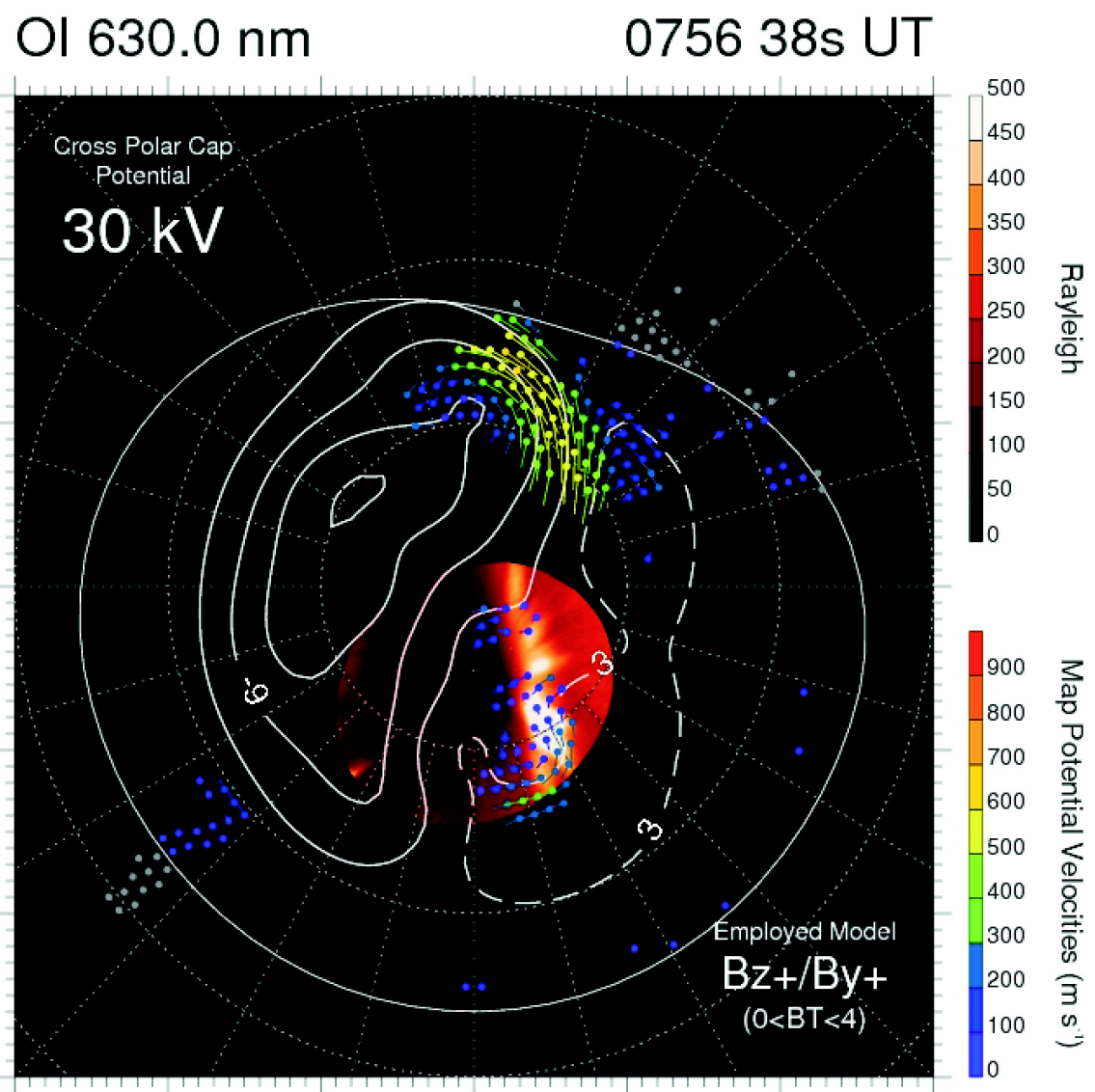

Fig. 8. Global map of plasma convection inferred from SuperDARM measurements at 07:56 UT and location of the optical form. Midnight is at the bottom.

data are organized in columns with the most left (right) one representing the beginning (end) of the interval. Such a presentation allows one to see the azimuthal position of the SA form within the radar FoV (and thus its duskward progression) and simultaneously to see the plasma flow component along each beam. Since the optical form was only slightly tilted with respect to the radar beams (Figs. 4 and 5), the velocities presented are almost the component of the plasma flow tangential to the optical form. There is some minor variation in the flow angle, as the azimuth of the beam changes, but in the central RANK beams, where the most of interesting data were collected, this effect is not significant, perhaps less than $\sim 10-20 \%$.

The first column of Fig. 7 shows the most dynamic part of the event when the optical form was quickly crossing the radar FoV. One can clearly see that the flow reversal is well "attached" to the western edge of the luminosity band and it shifts westward synchronously with the luminosity. The convection velocity in front of the luminosity edge is comparable in magnitude to the one behind it; the velocity magnitudes are of the order of $300 \mathrm{~m} / \mathrm{s}$ although some variations are discernible. For example, for the 07:54 UT frame, they are 330 (westward of the edge) and $300 \mathrm{~m} / \mathrm{s}$ (eastward of the edge) whereas for the 07:58 UT frame, they are -300 and $280 \mathrm{~m} / \mathrm{s}$. The first frame of the second column corresponds to the time when the SA form slowed its duskward progression and perhaps started splitting onto two bands; optical measurements do not allow clearly identify splitting as the forms were still away from the camera zenith. The velocity shows formation of a small dip in beam 6 in the antisunward portion of the arc-associated flow. This dip enhances later, see the 08:08 UT frame; at these times, a double 
structure of SA form is clearly recognizable in the optical data. The second column in Fig. 7 illustrates progression of the dip in the velocity and concurrent changes in the flow around the forms. We note that the velocity within the dip does not reach negative values (sunward flow). The last column presents measurements for the period when the SA form was leaving the RANK FoV. The 08:14 UT frame shows enhanced plasma flow at the dawnward edge of SA form and the last two frames at 08:16 and 08:18 UT show that as the SA form passed the radar FoV, the velocity decreased significantly and stayed at a level of $\sim 100 \mathrm{~m} / \mathrm{s}$ being towards the radar (antisunward direction). An interesting feature of the observations at $08: 12-08: 14$ UT is that during this period a significant portion of the SA form was covering the radar beams $0-4$ whose velocities were remarkably around zero. These signals were most likely the ground scattered echoes; they were received because the electron density inside the optical form was very high and thus provided significant radar wave bending at the bottom of the ionosphere, perhaps even at the E region heights.

\section{Global-scale convection pattern and SA form location and orientation}

Plasma flows associated with SA arcs and their role in the formation of the global convection pattern is one of important but unresolved so far issues (Zhu et al., 1997). For the considered event, only the Rankin Inlet and Hankasalmi SuperDARN radars collected reasonable amount of data and the assessment of the global convection pattern is rather limited. Standard SuperDARN convection maps, without the RANK data, show a two-cell convection pattern typical for $B_{z} \sim B_{y} \sim+3 \mathrm{nT}$. No special signatures of plasma flows in the dawn sector can be recognized as the pattern is driven by the statistical model of plasma convection.

Figure 8 shows a global 2-min convection map obtained by using all available velocity data at 07:56 UT along with the SA arc. Overall two-cell pattern is well recognizable but the dawn cell has the mesoscale structure with sheared flow associated with the arc.

Unfortunately, not much data is available to explore how this local convection cell is merged with the overall convection pattern for the entire event. This task is in our mind for other events that we hope to investigate as joint RANK-RES observations continue.

\section{Magnetometer perturbations related to the duskward moving auroral forms}

During the event under study, several magnetometers were conveniently located to study Earth magnetic field (current) changes associated with the SA form progression, Fig. 1. While investigating the magnetometer data, we kept in mind that the fundamental question as to whether magnetometers show signatures of SA arcs has not been resolved yet and this has been recognized as one of the outstanding issues (Zhu et al., 1997). In this view, our main goal is first to infer the magnetometer signatures of the SA arc and then to check if they are consistent with the radar data.

Figure 9 shows variations of the $\mathrm{X}, \mathrm{Y}$ and $\mathrm{Z}$ components of the Earth's magnetic field at four locations (Fig. 2), Resolute Bay (RES), Cambridge Bay (CBB), Taloyak (TALO), and Rankin Inlet (RANK). In selecting these sites, we recognized that all magnetometers are stretched roughly along the SA form, Figs. 1, 4 and 5. The CBB magnetometer is located slightly westward (duskward) of others, but this is actually an advantage as the magnetic effects slightly away from the SA form can be assessed. In Fig. 9 we indicated by a vertical bar the period during which the SA form was roughly located near the zenith of the RES magnetometer.

Figure 9 shows clear perturbations associated with the SA form progression at RES and TALO, in all three components. This is not a surprise because the SA form crossed the zenith of each of these magnetometers. At CBB, the magnetic perturbations were similar to the ones at RES and TALO but with some delay which is what is expected taking into consideration the fact that the SA form reached longitudes of CBB $\sim 3-5$ min later. At RANK, the magnetic signatures were not so clear with the exception of the $\mathrm{Z}$ component. This is probably because the SA form was not so much extended equatorward, consistent with what we have inferred from the radar data alone (Sect. 7).

In the $\mathrm{Z}$ component, one can see a negative "bay" for the period prior to the SA form arrival to the zenith and positive "bay" after it passed the zenith. Similar perturbations in the $\mathrm{Z}$ component were detected by other magnetometers (bottom panels of Fig. 9). These perturbations can be interpreted in terms of an equivalent string-like current source that is aligned with the optical form and that has the current flow towards noon (antisunward convection). With time, the source shifts duskward, synchronously with the form.

In the Y component, there were negative bays both prior and after the SA form zenith location. To interpret these perturbations, one can assume that the single-string current was actually an extended one in the direction perpendicular to the string and that it actually was split onto two components, with a stronger filament current flowing towards noon (antisunward convection) and a weaker filament current flowing towards midnight (sunward convection). For a magnetometer location between these two filaments, the perturbation in the Y component would be decreased as compared to the perturbations for magnetometer locations away from the zenith. At far locations, effect of the weaker filament is overpowered by the effect of the stronger filament, and only negative bays are seen. Such a model of the current source would be consistent with the radar detection of splitting of the antisunward portion of the flow onto two components occurred simultaneously with the SA arc splitting (Figs. 1 and 7). 


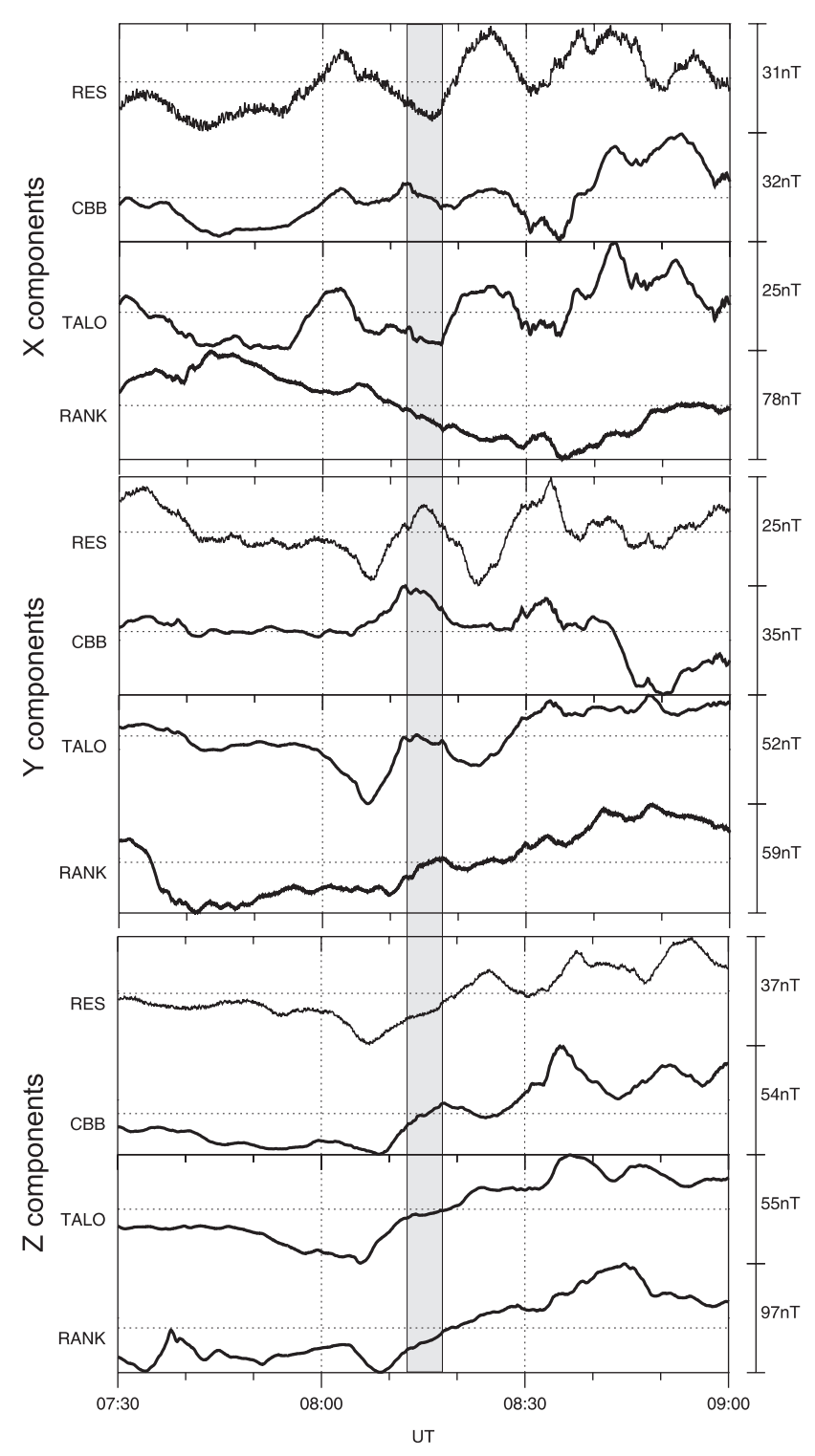

Fig. 9. $\mathrm{X}, \mathrm{Y}$ and $\mathrm{Z}$ components (geographic coordinates) of the Earth magnetic field fluctuations recorded at Resolute Bay (RES), Cambridge Bay (CBB), Taloyak (TALO) and Rankin Inlet (RANK) between 07:30 and 09:00 UT on 15 February 2007.

Perturbations in the $\mathrm{X}$ component are consistent with the double-filament model with a stronger dawnside filament. Indeed, Fig. 9 shows positive deflections for the magnetometer locations both westward (duskward) and eastward (dawnward) of the optical form. For the magnetometer locations near the zenith (under the optical form), the magnetic deflection was small (perhaps the background value was reached), implying that the magnetic effects of individual sub-filaments were compensating each other. We should note that although the magnetometer data can be interpreted in terms of simple double-filament model, why one of the filaments was stronger is not very clear. Perhaps the fila- ments were different in the width and their extension along the arc. The domination of the dawnside filament string is not a surprise because the electron density should be larger on the dawnside of the SA form moving duskward (Crain et al., 1993) and this was flagged in the radar data by larger elevation angles on this side of the form.

An important conclusion from the above magnetometer data consideration is that although signatures of the SA form are well seen for the magnetometers whose zenith was passed by the SA form, derivation of the true convection structure from the magnetometer data alone is not a simple task.

\section{Discussion and conclusions}

In this study, the Rankin Inlet SuperDARN HF radar measurements were combined with all-sky camera observations at Resolute Bay to investigate radar signatures of, and the plasma flows around, the sun-aligned optical form originated on the dawnside, probably near the auroral oval, and moving across the polar cap, thus entering the FoV of both the radar and the all-sky camera. The optical form was stretched roughly along the radar's central beams so that the component of the plasma flow tangential to the arc was monitored.

Significant difference of the presented here HF radar observations of SA arc as compared to previous cases of theta aurora (Chang et al., 1998; Milan et al., 2005; Liu et al., 2005; Eriksson et al., 2006) and auroral zone evening arcs (Uspensky et al., 2001) is that the echoes were detected continuously and in most of the space around the optical structure at radar ranges of $<900 \mathrm{~km}$. In the past, patchy and episodic echo occurrence has been enough to infer an average convection pattern associated with theta-aurora, an optically more intense event. We should note that many features that have been described here, were also observed during another minor SA form event happened just $\sim 10$ min prior to the main event. The intensity of luminosity for this event was very low so that the form was barely discernible from the optical data, but the radar data showed clear signatures in echo power ("gap" at the form's location) and velocity (velocity reversal near the form location) and in fact, the event was first identified in the radar data and only later optical signatures were found. Other SA events on 15 February 2007 showed radar signatures in the velocity, and these are currently under investigation.

Formulation of the radiophysical model for the echo signal formation in the considered event is a challenging task. Range resolution of the RANK measurements is $45 \mathrm{~km}$ while the thickness of the individual auroral forms is expected to be less than that. We believe that signal integration effect was one of the reasons why the echo power did not drop significantly when the arc was within a specific radar gate. VHF and UHF measurements near the intense auroral oval arcs, having better spatial resolution, show stronger effect (Uspensky et al., 1983). Another expected feature of echoes in the 
vicinity of an auroral arc is the velocity enhancement in the area adjacent to the arc (Uspensky et al., 1983; Timofeev et al., 1987; Aikio et al., 2002). We did not find any evidence for the effect in Rankin data; this also can be explained by limitations in the radar spatial resolution. We note that another possible reason for the weaker effect is that the polar cap arcs are not so intense as the ones in the auroral zone.

Additional challenge for understanding the HF signal formation from SA arcs comes from the fact that HF radar waves experience enormous amount of refraction while passing an optical arc as the electron densities achievable within the polar cap arc can be significant (up to $2 \times 10^{5} \mathrm{~cm}^{-3}$, Mende et al., 1988). We have identified (Sect. 7) a short period when strong refraction provided detection of ground scattered signals at ranges as short as $540 \mathrm{~km}$ (gate 8, Fig. 7, frame 08:12 UT). Presence of the strong arc-associated electron densities and their horizontal gradients might lead to lateral bending of radio waves and echo reception from the "walls" of an arc instead of from inside it. Another complicating factor is the strong height variation of the electron density profiles related to SA arcs; these were predicted theoretically by Crain et al. (1993). The radar data presented support one major conclusion of the modeling by Crain et al. (1993) that the electron density is enhanced on the dawnside of a duskward moving SA arc; the effect was flagged by strongly increased elevation angles of echo arrival from the dawnside of the arc.

In passing by, we note that strong variations of the radar echo height might be responsible for some misalignment of radar and optical data. We have compared optical and radar maps by assuming various heights for the luminosity, from $220 \mathrm{~km}$ to $300 \mathrm{~km}$, and for fixed echo height of $300 \mathrm{~km}$. We found that mutual displacements are noticeable but not drastic. For this reason we selected for the presentation the height of $250 \mathrm{~km}$. More significant changes might occur if the echo heights are below $200 \mathrm{~km}$ or above $300 \mathrm{~km}$, which potentially may occur (Koustov et al., 2007).

Another argument for the extreme significance of ionospheric HF refraction for the event under consideration is the fact that the echoes were detected only at relatively short ranges of $<1000 \mathrm{~km}$. The arc was quite extended latitudinally, and the flow was fast $(\sim 300 \mathrm{~m} / \mathrm{s})$, so that one expects that the conditions for the ionospheric plasma instabilities, e.g. gradient-drift instability, were satisfied in much more significant area. Ionospheric refraction thus provided focusing of radio waves only at certain ranges.

Clearly, to understand HF echo formation in the vicinity of the polar cap SA arcs, detailed information on the vertical and horizontal distribution of the electron density is required. This can be achieved in future, with the installation of the incoherent scatter radar at Resolute Bay.

In terms of general morphology, the observed SA form was a typical SA feature reported in the past. It was first clearly seen when the IMF $B_{z}$ was positive and $B_{y}$ was negative, and the arc's motion was taking place while $B_{y}$ was changing polarity towards positive values. Originally, the arc was moving with the speed of $\sim 200 \mathrm{~m} / \mathrm{s}$, but slowed down and stopped, perhaps in response to IMF $B_{z}$ decrease to almost zero at the end of the discussed period. There were two subsequent reactivations of arc's duskward motion, all started with a changing IMF $B_{y}$ to larger positive values (while $B_{z}$ being positive).

While moving duskward, the SA form experienced splitting onto two components. Similar phenomenon but for a duskside transpolar arc has been investigated recently by Eriksson et al. (2006). The IMF conditions for their event were different, and direct comparison with our observations does not make sense. However, an important component of their work is an electrodynamical model of the currents associated with the split arc. Eriksson et al. (2006) suggested that the split transpolar arc electrodynamics is governed by two upflowing field-aligned currents (FAC) co-locating with the arcs and an additional downward FAC located in between them. The FACs are interconnected through Pedersen currents in the ionosphere so that, for both arcs, the electric field is converging, i.e. directed towards the center of each upward FACs.

Our data at the beginning (end) of the event, when a single SA form was observed, do show a converging electric field on the dusk side (dawn side) of the arc. Also, the flow was quite uniform duskward and downward the SA form location, along the arc extension $(\sim 400 \mathrm{~km}$, from $400 \mathrm{~km}$ to $800 \mathrm{~km}$, Fig. 5) which is remarkable and quite different from observations by Gallaher (1997) and Mende et al. (1988).

As the SA form was evolving (or seen as) into two component structure, the radar measurements are more difficult to interpret. The duskward edge of the arcs was all time co-locating with the large-scale flow reversal, and the arc splitting occurred within the antisunward portion of the flow shear. We do not have direct evidence for the onset of the sunward flows in the area between the split arcs, as only an overall decrease (dip) of the velocity was detected (e.g. 08:08 UT frame of Fig. 7). However, we can interpret the flow velocity decrease in the area between the arcs as the onset of additional sunward $\boldsymbol{E} \times \boldsymbol{B}$ flow "carving out" velocity from the overall antisunward flow. To be consistent with the model by Eriksson et al. (2006) we have to assume that this sunward flow between the split arc components consisted of sunward and antisunward components with the sunward one being stronger. This suggestion would be consistent with our magnetometer signatures of the SA form, as a magnetometer cannot resolve two current filaments separated by $\sim 100 \mathrm{~km}$ and would sense a large-scale antisunward flow and an "effective" sunward flow channel located between the split arcs. In terms of the radar velocity data, unfortunately, we have to keep in mind that our measurements may not have required spatial resolution as the arc separation was of the order of $\sim 100-150 \mathrm{~km}$ from the optical data and the velocity peaks (Fig. 7, 08:08 UT frame) were separated by $\sim 5$ beam positions which also gives separation of the order of $150 \mathrm{~km}$ 
$\left(3.24^{\circ} \times 5=16.2^{\circ}\right.$ of azimuthal separation which gives $150 \mathrm{~km}$ at the radar range of $540 \mathrm{~km}$ ). Such separation is not much more than the radar beamwidth in the azimuthal direction.

In terms of convection on a global scale, the SA form looked as a separate entity that, while moving, was perturbing the general two-cell convection pattern on the dawnside. Arc-associated shear flow did not coincide with the reversal of global-scale convection cell as mentioned by Zhu et al. (1997). We are not confident in these two conclusions, however, as the SuperDARN data were very limited.

The data available do not allow us to say definite words on the magnetospheric origin of the observed SA forms. While the forms were moving duskward, the remnants of the auroral oval luminosity at the eastern edge of the camera FoV were identifiable. This suggests that the arc was formed by splitting/detaching from the oval and not by protruding into the polar cap from the midnight auroral oval. Thus, processes of merging between the IMF and Earth's magnetic field (e.g. Blomberg et al., 2005) are more likely responsible for the arc formation and dynamics as compared to the evolution of interchange instabilities (e.g. Golovchanskaya et al., 2006). Sojka et al. (1994) proposed the coupled magnetosphereionosphere model that considers a shear Alfven wave interacting with the ionosphere. One of their predictions is that for the background convection velocities of $>15 \mathrm{mV} / \mathrm{m}$, the wave would lead to a splitting of the initial conductance band onto fine structures and multiple arcs should be observed. In our event, the background convection was probably below this threshold, but arc-associated convection flows were very close to the threshold. Although splitting onto two arcs did occur, the arc did not continue spreading apart, as the model would expect, but rather stayed at the fixed separation of $\sim 150 \mathrm{~km}$.

Major results of the present study can be formulated as follows:

1. A case of HF radar continuous monitoring of SA form moving duskward is presented. Observations were performed in the dawn sector at magnetic latitudes of $\sim 77^{\circ}$. Radar signatures of the moving SA form have been identified as 1) a significant echo power drop spatially coinciding with the luminosity duskside boundary at the F-region heights, 2) a significant increase in plasma flow magnitude and echo spectral width duskward and dawnward of the form, 3) a sharp reversal of the plasma flow from sunward to anti-sunward near the structure's duskside (at the F-region heights) and 4) a strong increase of the elevation angle of echo arrival in the wake of the moving form.

2. Magnetic perturbations associated with the SA form passing the zenith of a magnetometer have been identified. For a magnetometer location to the west (duskward) or to the east (dawnward) of the arc the $\mathrm{X}$ component shows a positive deflection of $\sim 15-20 \mathrm{nT}$ while the $\mathrm{Y}$ component shows negative deflection of a comparable magnitude. The $\mathrm{Z}$ component shows a negative (positive) deflection for the magnetometer location to the west (east) of the SA form. Radar data indicate that it is hardly possible to infer the structure of the convection around the SA form on the basis of magnetometer data alone.

3. SuperDARN network global pattern estimates indicate that the local convection reversal associated with the SA form was not aligned/coinciding with the convection reversal of global-scale convection cells; rather, it was located poleward (duskward) of it. The duskward progression of the arc-associated reversal and optical arc were probably reflecting the mesoscale restructuring of the global-scale convection pattern from $B_{y}-$ to $B_{y}+$ configurations.

Acknowledgements. This work was supported by Grants-in Aid for Scientific Research (16403007; 19340141; 19403010; 20244080) from the Ministry of Education, Culture, Sports, Science and Technology of Japan, and by Project 2 of the Geospace Research Center, Solar Terrestrial Environment Laboratory. The PolarDARN HF radar at Rankin Inlet was initially funded by the Canada Foundation for Innovation NSERC Grant to J.-P. St-Maurice (Univ. of Saskatchewan) and is currently operated by the Univ. of Saskatchewan radar group with funding from the Canadian Space Agency and NSERC to G. J. Sofko. A. V. Koustov acknowledges the Solar-Terrestrial Environment Laboratory of Nagoya University for funding during his stay in Japan. The work was also supported by NSERC grant to A. V. Koustov. ACE data on the IMF and solar wind provided by N. Ness (Bartol Research Institute) through CDAWEB are acknowledged. Magnetometer data provided by the Canadian Hazard Information Canter (Ottawa) and CARISMA project of the Canadian Geospace Monitoring Program (Canadian Space Agency) are acknowledged.

Topical Editor M. Pinnock thanks two anonymous referees for their help in evaluating this paper.

\section{References}

Aikio, A. T., Lakkala, T., Kozlovsky, A., and Williams, P. J. S.: Current systems of stable drifting auroral arcs in the evening sector, J. Geophys. Res., 107, 1424, doi:10.1029/2001JA009172, 2002.

Blomberg, L. G., Cumnock, J. A., Alexeev, I. I., Belenkaya, E. S., Bobrovnikov, S. Yu., and Kalegaev, V. V.: Transpolar aurora: time evolution, associated convection patterns, and a possible cause, Ann. Geophys., 23, 1917-1930, 2005, http://www.ann-geophys.net/23/1917/2005/.

Carlson Jr., H. C., Heelis, R. A., Weber, E. J., and Sharber, J. R.: Coherent mesoscale convection patterns during northward interplanetary magnetic field, J. Geophys. Res., 93, 14 501-14 514, 1988.

Chang, S.-W., Scudder, J . D., Sigwarth, J. B., Frank, L. A., Maynard, N. C., Burke, W. J., Peterson, W. K., Shelley, E. G., Friedels, R., Blake, J. B., Greenwald, R. A., Lepping, R. P., Sofko, G. J., Villain, J.-P., and Lester, M.: A comparison of a model for the theta aurora with observations from Polar, Wind, and SuperDARN, J. Geophys. Res., 103, 17 367-17390, 1998. 
Crain, D. J., Sojka, J. J., Schunk, R. W., and Zhu, L.: Parametrized study of the ionospheric modification associated with sunaligned polar cap arcs, J. Geophys. Res., 98, 6151-6152, 1993.

Danskin, D. W., Koustov, A. V., Ogawa, T., Nishitani, N., Nozawa, S., Milan, S. E., Lester, M., and Andre, D.: On the factors controlling occurrence of F-region coherent echoes, Ann. Geophys., 20, 1385-1397, 2002, http://www.ann-geophys.net/20/1385/2002/.

Eriksson, S., Provan, G., Rich, F. J., Lester, M., Milan, S. E., Massetti, S., Gosling, J. T., Dunlop, M. W., and Rème, H.: Electrodynamics of a split-transpolar aurora, J. Geophys. Res., 111, A11319, doi:10.1029/2006JA011976, 2006.

Gallagher Jr., H. A.: Radar and optical observations of plasma convection associated with very high-latitude auroral arcs, $\mathrm{PhD}$ Thesis, Boston College, USA, 1997.

Golovchanskaya, I. V., Kullen, A., Maltsev, Y. P., and Biernat, H.: Ballooning instability at the plasma sheet-lobe interface and its implications for polar arc formation, J. Geophys. Res., 111, A11216, doi:10.1029/2005JA011092, 2006.

Hultqvist, B.: The aurora, in: Handbook of the solar-terrestrial environment, edited by: Kamide, Y. and Chian. A., Springer-Verlag Berlin Heidelberg, pp. 334-354, doi:10.1007/11367758_13, 2007.

Koustov, A. V., Danskin, D. W., Makarevitch, R. A., and Gorin, J. D.: On the relationship between the velocity of E-region HF echoes and $\boldsymbol{E} \times \boldsymbol{B}$ plasma drift, Ann. Geophys., 23, 371-378, 2005 , http://www.ann-geophys.net/23/371/2005/.

Koustov, A. V., Danskin, D. W., Uspensky, M. V., Ogawa, T., Janhunen, P., Nishitani, N., Nozawa, S., Lester, M., and Milan, S.: Velocities of auroral coherent echoes at 12 and $144 \mathrm{MHz}$, Ann. Geophys., 20, 1647-1661, 2002,

http://www.ann-geophys.net/20/1647/2002/.

Kozlovsky, A., Nilsson, H., Sergienko, T., Aikio, A. T., Safargaleev, V., Turunen, T., and Kauristie, K.: On the field-aligned currents in the vicinity of prenoon auroral arcs, Geophys. Res. Lett., 32, L18104, doi:10.1029/2005GL023120, 2005.

Kozlovsky A., Aikio, A., Turunen, T., Nilsson, H., Sergienko, T., Safargaleev, V., and Kauristie, K.: Dynamics and electric currents of morningside Sun-aligned auroral arcs, J. Geophys. Res., 112, A06306, doi:10.1029/2006JA012244, 2007.

Kullen, A., Brittnacher, M., Cumnock, J. A., and Blomberg, L. G.: Solar wind dependence of the occurrence and motion of polar auroral arcs: A statistical study, J. Geophys. Res., 107(A11), 1362, doi:10.1029/2002JA009245, 2002.

Liou, K., Ruohoniemi, J. M., Newell, P. T., Greenwald, R., Meng, C.-I., and Hairston, M. R.: Observations of ionospheric plasma flows within theta auroras, J. Geophys. Res., 110, A03303, doi:10.1029/2004JA010735, 2005.

Makarevitch, R. A., Honary, F., and Koustov, A. V.: Simultaneous measurements of E- and F-region Doppler velocities at large flow angles, Ann. Geophys., 22, 1177-1185, 2004, http://www.ann-geophys.net/22/1177/2004/.

Mende, S. B., Doolittle, J. H., Robinson, R. M., and Vondrak, R. R.: Plasma drifts associated with a system of sun-aligned arcs in the polar cap, J. Geophys. Res., 93, 256-264, 1988.
Milan, S. E., Hibert, B., and Grocott, A.: Formation and motion of a transpolar arc in response to dayside and nightside reconnection, J. Geophys. Res., 110, A01212, doi:10.1029/2004JA010835, 2005.

Nilsson, H., Kozlovsky, A., Sergienko, T., and Kotikov, A.: Radar observations in the vicinity of pre-noon auroral arcs, Ann. Geophys., 23, 1785-1796, 2005, http://www.ann-geophys.net/23/1785/2005/.

Obara, T., Mukai, T., Hayakawa, H., Tsuruda, K., Matsuoka, A., Nishida, A., Fukunishi, H., Zhu, L., Sojka, J., and Crain D.: Multiple polar cap arcs: Akebono (Exos D) observations, Radio Sci., 31, 645-654, 1996.

Robinson, R. M., Vondrak, R. R., and Friis-Christensen, E.: Ionospheric currents associated with a sun-aligned arc connected to the auroral oval, Geophys. Res. Lett., 14, 656-659, 1987.

Robinson, R. M. and Mende, S.: Ionization and electric field properties of auroral arcs during magnetic quiescence, J. Geophys. Res., 95, 21 111-21 121, 1990.

Sojka, J. J., Zhu, L., Crain, D. J., and Schunk, R. W.: Effect of highlatitude ionospheric convection on Sun-aligned polar cap arcs, J. Geophys. Res., 99(N5), 8851-8863, 1994.

Shiokawa, K., Yumoto, K., Hayashi, K., Oguti, T., and McEwen, D. J.: A statistical study of the motions of auroral arcs in the highlatitude morning sector, J. Geophys. Res., 100, 21 979-21 985, 1995.

Shiokawa, K., Ogino, T., Hayayshi, K., and McEwen, D. J.: Quasiperiodic poleward motions of morning side Sun-aligned arcs: A multi event study, J. Geophys. Res., 102, 24 325-24 332, 1997.

Shiokawa, K., Katoh, M., Satoh, M., Ejiri, M. K., Ogawa, T., Nakamura, T., Tsuda, T., and Wiens, R. H.: Development of optical mesosphere thermospheric imagers (OMTI), Earth, Planets, Space, 31, 887-896, 1999.

Timofeev, E. E., Vallinkoski, M. K., Kozelova, T. V., Yahnin, A. G., and Pellinen, R. J.: Systematics of arc-associated electric fields and currents as inferred from radar backscatter measurements, J. Geophys., 61, 122-137, 1987.

Uspensky, M. V., Eglitis, P., Opgenoorth, H., Starkov, G., Pulkkinen, T., and Pellinen, R.: On auroal dynamics observed by HF radar: 1. Equatorward edge of the afternoon-evening diffuse luminosity belt, Ann. Geophys., 18, 1560-1575, 2001, http://www.ann-geophys.net/18/1560/2001/.

Uspensky M. V., Pellinen, R. J., Baumjohann, W., Starkov, G. V., Nielsen, E., Sofko, G., and Kaila, K. U.: Spatial variations of ionospheric conductivity and radar auroral amplitude in the eastward electrojet region during pre-substorm conditions, J. Geophys., 52, 40-48, 1983.

Valladares, C. E., Carlson Jr., H. C., and Fukui, K.: Interplanetary magnetic field dependency of stable Sun-aligned polar cap arc, J. Geophys. Res., 99, 6247-6272, 1994.

Valladares, C. E. and Carlson Jr., H. C.: The electrodynamic, thermal, and energetic character of intense Sun-aligned arcs in the polar cap, J. Geophys. Res., 96, 1379-1400, 1991.

Zhu, L., Schunk, R. W., and Sojka, J. J.: Polar cap arcs: a review, J. Atmos. Terr. Phys., 59, 1087-1126, 1997.

Zhu, L., Valladares, C. E., Sojka, J. J., Schunk, R. W., and Crain, D. J.: Model-observation comparison of multiple polar cap arcs, J. Geophys. Res., 101, 323-333, 1996. 